(C) 2021 IEEE. Personal use of this material is permitted. Permission from IEEE must be obtained for all other uses, in any current or future media, including reprinting/republishing this material for advertising or promotional purposes, creating new collective works, for resale or redistribution to servers or lists, or reuse of any copyrighted component of this work in other works. 


\title{
Assessment of Validity Conditions for Black-Box EMI Modelling of DC/DC Converters
}

\author{
Lu Wan, Abduselam Beshir, Xinglong Wu, Xiaokang Liu, Flavia Grassi, Giordano Spadacini, Sergio Pignari \\ Dept. of Electronics, Information and Bioengineering (DEIB) \\ Politecnico di Milano \\ Milan, Italy \\ \{lu.wan, abduselamhamid.beshir, xinglong.wu, xiaokang.liu, flavia.grassi, giordano.spadacini, sergio.pignari\}@polimi.it
}

\begin{abstract}
Black-box modelling approaches, based on suitable sets of measurements at the output ports of the device, are often exploited for the modelling of power converters to predict their conducted emissions. However, these techniques can be effectively applied only if the device to be modelled can be approximately treated as a linear and time-invariant (LTI) system. This assumption is not necessarily satisfied by every power converter. In order to investigate suitable conditions assuring effectiveness of black-box modelling for a boost converter, this work investigates the role that the converter input capacitors and the functional inductor play in masking the inherent non-linear and time variant behavior of the switching modules, and their impact on the effectiveness of the proposed black-box model. It will be shown that preliminary measurements of the differential mode impedance of the converter can provide useful information on the feasibility of black-box modelling techniques, even in the absence of detailed information on the internal architecture of the converter.
\end{abstract}

Keywords-Black-box modelling, Boost converters, Common mode and Differential mode noise, Conducted emissions.

\section{INTRODUCTION}

The wide diffusion of power converters in many sectors, such as transportation and advanced metering infrastructures, gives rise to unexpected conducted emissions which lead to coexistence issues between power and data lines [1]. With the aim of reducing emissions, accurate electromagnetic interference (EMI) models of power converters are keyingredients for predicting the actual noise levels, and thus designing suitable mitigating solutions like optimized EMI filters and/or alternative modulation strategies.

EMI modelling strategies available for power converters can be mainly classified into two types: circuit models and behavioral models [2]. Circuit models should include not only the functional behavior of the converter but also additional circuit elements representing parasitic coupling phenomena, whose inclusion is of paramount importance for electromagnetic compatibility analyses. Drawbacks of such a modelling approach are (a) difficult identification of the values of parasitic components; and (b) possible nonconvergence issues in time-domain simulations, especially when many switches are present in the system [3]. Due to these reasons, resorting to such a modelling approach is reasonable for simple converters only. Conversely, behavioral models, which treat the converter under analysis as a black box, look more feasible to the purpose, as they are obtained by a set of measurements carried out at the external ports of the converter [4]. Hence, this technique looks especially beneficial for complex converters and/or commercial converters. Several examples of black-box models developed for EMI prediction of commercial converters can be found in

This project has received funding from the European Union's Horizon 2020 research and innovation programme under the Marie Skłodowska-Curie grant agreement No 812753. the technical literature [5-7], which prove the accuracy of this technique.

In general, black-box modelling resorts to an equivalent representation of the converter in terms of Thevenin or Norton equivalent circuits, which is aimed at reproducing the frequency response of the converter at the input/output ports in spite of the specific loading conditions. However, blackbox modelling is applicable only if the system under analysis can be approximately considered as a LTI system. It is observed that, although power converters are non-linear and time variant systems, however the presence of EMI input filters or decoupling capacitors can often mask the switching behaviors, so that the LTI assumption can be considered to be approximately satisfied in many converters [8-10].

However, this conclusion cannot be generalized to all converters without further investigations. Indeed, some recent works [11], [12] show that differently from the common mode (CM) impedance, which is mainly determined by the effects due to parasitics, the differential mode (DM) impedance may exhibit not-negligible sensitivity to the operating conditions. Therefore, extracting the black-box model of the converter under certain operating conditions does not assure model effectiveness for predicting the conducted emissions in other operating conditions. Hence, a preliminary investigation on the conditions assuring model effectiveness is required.

In line with this objective, this work investigates suitable conditions for deriving black-box models of commercial boost converters, with particular focus on the assumption of linearity and time invariance. To this end, the procedure proposed in [6] for extracting black-box model parameters is employed, and the role played by the presence of input capacitors and the functional inductor in masking the non-linear and time variant behavior of the converter is investigated. The analysis is also extended to investigate the characteristics of the frequency response of the differential mode (DM) impedance. As a matter of fact, since previous investigations [11], [12] have proved its possible sensitivity to the operating conditions, measurement of the DM impedance under different operating conditions can provide useful hints to assess the feasibility of black-box modelling of commercial converters, for which detailed information on the internal architecture is not always available.

\section{STRUCTURE UNDER ANALYSIS AND CIRCUIT MODELS}

The $14 \mathrm{~V} / 42 \mathrm{~V}$ boost converter with input capacitors presented in [13] is considered as the system under analysis. This converter is for automotive applications where these two voltage levels are commonly used. The functional circuit model of the converter fed by a $14 \mathrm{Vdc}$ battery is shown in Fig. 1. The switching frequency is $100 \mathrm{kHz}$ and the duty cycle is 0.75 . The complete circuit model with parasitic components 


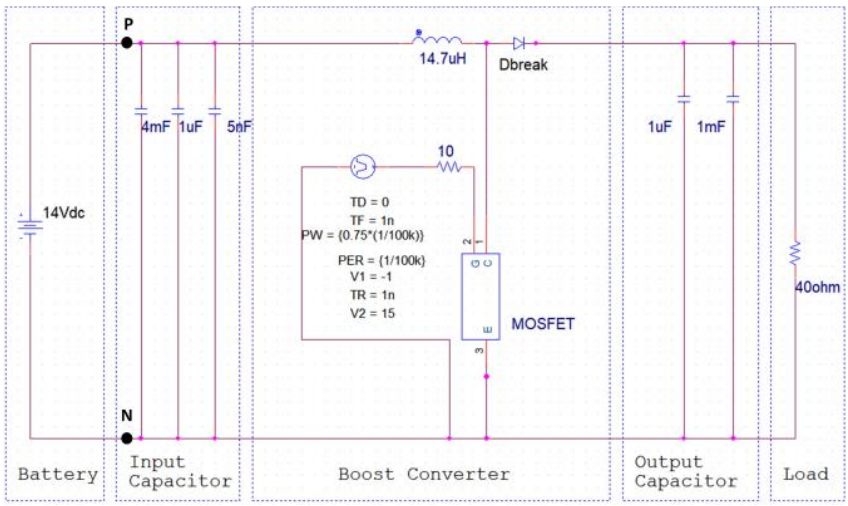

Fig. 1. Circuit model (functional part) of the system under analysis.

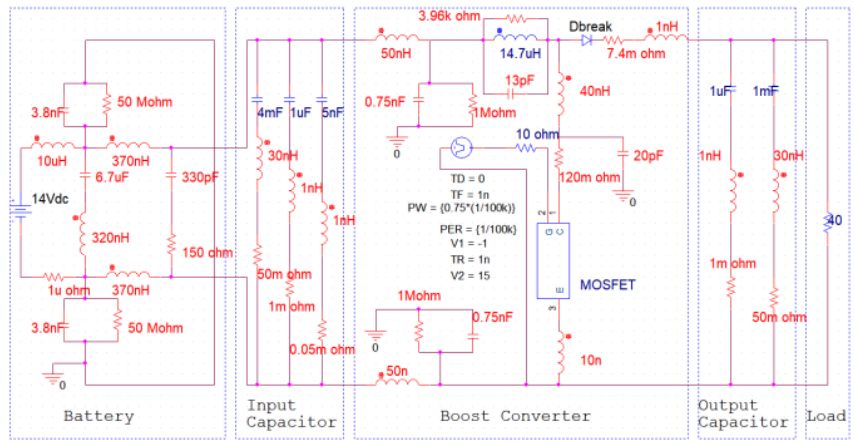

Fig. 2. Circuit model of the system under analysis including parasitic components.

is highlighted by red color in Fig. 2. They include: The equivalent series inductors and resistors of the input and output capacitors, parasitic parallel resistor and capacitor of functional inductors, parasitic components of the diode, and equivalent capacitors between the MOSFET and the heat sink, [13], [14]. Also, the high-frequency model of the battery is determined by including parasitic components between phase, neutral, and ground [15].

\section{BLACK-Box MODELLING PROCEDURES}

The experimental black-box modelling procedure proposed in [6] is here mimicked by SPICE simulations, which are best suited to the aims of the proposed investigation. Indeed, simulations rely only on the fundamental circuit properties of interest (non-linearity, time variance, parasitics, etc.) unveiling subtle effects that may be masked by measurement-uncertainty issues normally involved in experimental observations. As shown in the top panel of Fig. 3 , the converter is modelled at the output ports by an impedance matrix $\boldsymbol{Z}_{\text {dut }}$, representative for its passive part, and two current sources $I_{\mathrm{s} 1}$ and $I_{\mathrm{s} 2}$, representative for the active part.

Model parameters are identified in two steps. In the first step, the entries of matrix $\boldsymbol{Z}_{\text {dut }}$ are identified by $S$-parameter evaluation of the ports of the converter switched off. To this end, S-parameter measurement blocks [16] are included in the SPICE schematic to mimic a Vector Network Analyzer (VNA), and the $2 \times 2$ S-parameter matrix $S_{\text {dut }}$ of the converter is evaluated (see middle panel in Fig. 3). The impedance matrix $\boldsymbol{Z}_{\mathrm{dut}}$ of the converter is then calculated by

$$
\boldsymbol{Z}_{d u t}=R_{0}\left(\mathbf{1}_{2}+\boldsymbol{S}_{d u t}\right)\left(\mathbf{1}_{2}-\boldsymbol{S}_{d u t}\right)^{-1}
$$

where $R_{0}=50 \Omega$ is the standard impedance for $S$-parameter measurement; and $\mathbf{1}_{2}$ denotes the $2 \times 2$ unit matrix.
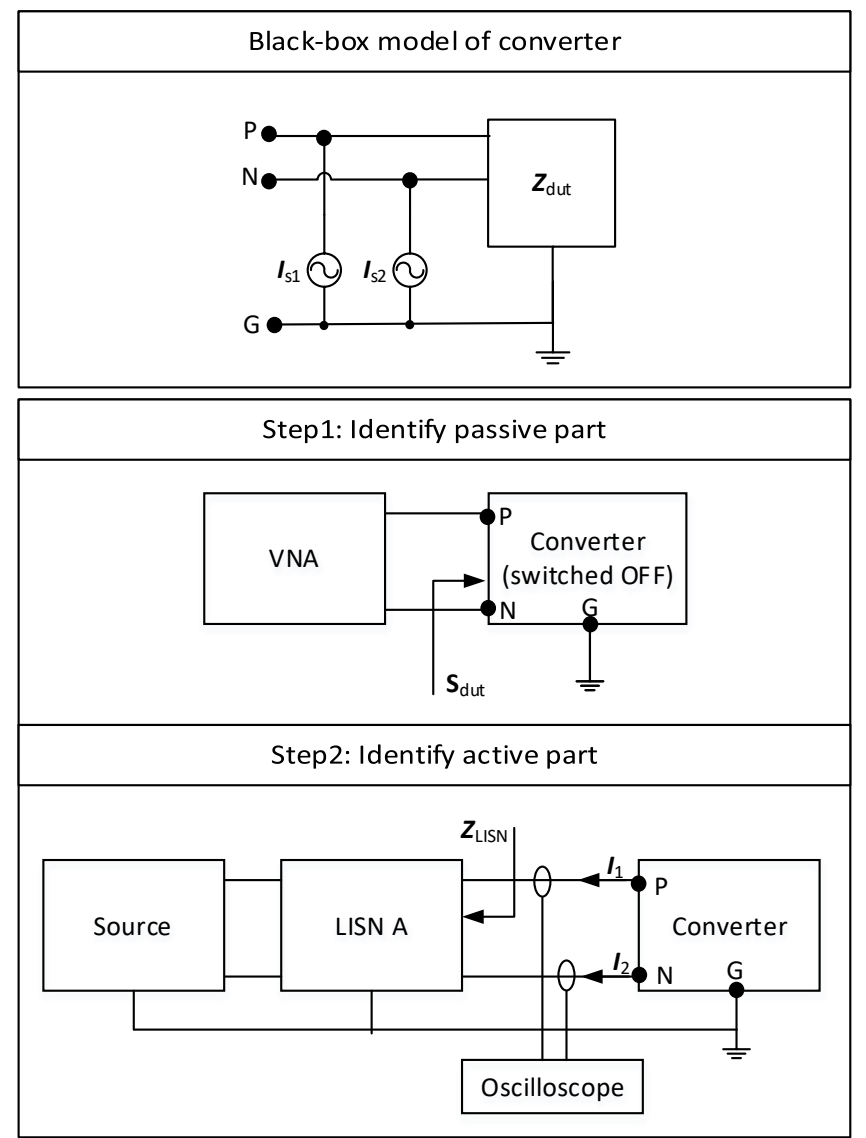

Fig. 3. Black-box model of the converter (top panel) and setups exploited for the identification of model-parameters (middle and bottom panel).

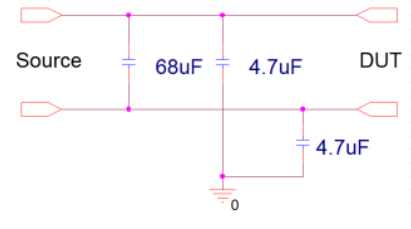

(a)

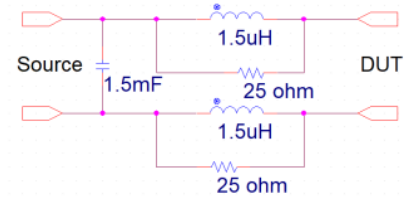

(b)
Fig. 4. (a) LISN A and (b) LISN B used for extraction and validation of black-box model parameters, respectively.

In the second step (see bottom panel in Fig. 3), the active part of the model is evaluated. To this, time-domain simulation of the conducted emissions exiting the boost converter were evaluated by connecting a Line Impedance Stabilization Network (LISN) at the output terminals. More specifically, the LISN shown in Fig. 4(a) is used, and the currents in the phase and neutral lines $\left(I_{1}\right.$ and $\left.I_{2}\right)$ are evaluated in the time domain, and converted into frequency domain. The equivalent current sources $I_{\mathrm{s} 1}$ and $I_{\mathrm{s} 2}$ are afterwards calculated by

$$
\left[\begin{array}{l}
I_{s 1} \\
I_{s 2}
\end{array}\right]=\left(\mathbf{1}_{2}+\boldsymbol{Z}_{d u t}^{-1} \boldsymbol{Z}_{L I S N}\right)\left[\begin{array}{l}
I_{1} \\
I_{2}
\end{array}\right]
$$

where $\boldsymbol{Z}_{\text {LISN }}$ denotes the $2 \times 2$ impedance matrix of the LISN evaluated at the DUT ports.

The second LISN shown in Fig. 4(b) will be exploited afterwards for model validation, that is to generate a different set of operating conditions to verify the effectiveness of the extracted black-box model. 


\section{EMI PREDICTIONS}

Once the parameters of the black-box model have been extracted by exploiting LISN A, the complete SPICE circuit model of the boost converter with LISN B in Fig. 4(b) is simulated in the time domain. The frequency response of the obtained phase current is then evaluated by Fast Fourier Transform [see blue spectrum in Fig. 5(a)], and compared with the corresponding predictions obtained by exploiting the black-box model derived in Section III.

The comparison is shown in Fig. 5(a), where the red dots represent the conducted emissions of the converter (evaluated at the switching frequency of $100 \mathrm{kHz}$ and all its harmonics up to $30 \mathrm{MHz}$ ) obtained by exploiting the black-box model. The satisfactory agreement between the two spectra indicates that the black-box model is quite accurate at almost every harmonic frequency, with only small discrepancies around 25 $\mathrm{MHz}$ due to the presence of a resonance. This resonance may be due to the interaction between the LISN B and the converter in the verification setup and it was not observed in the setup exploiting LISN A.

In order to investigate the role of the presence of the input capacitor(s), this component(s) was removed from Fig. 2. The procedure of derivation of the black-box model was repeated, as well as the aforesaid simulations. The comparison between the obtained current spectra is shown in Fig. 5(b).

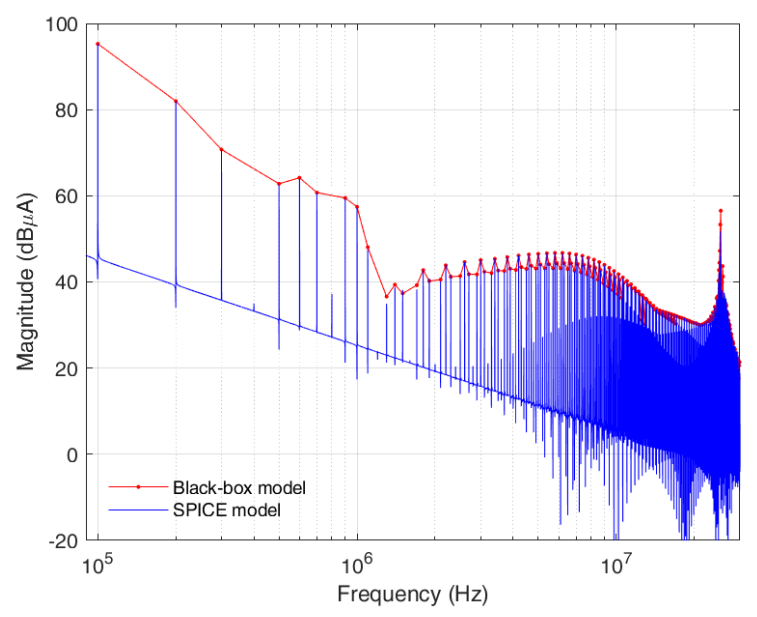

(a)

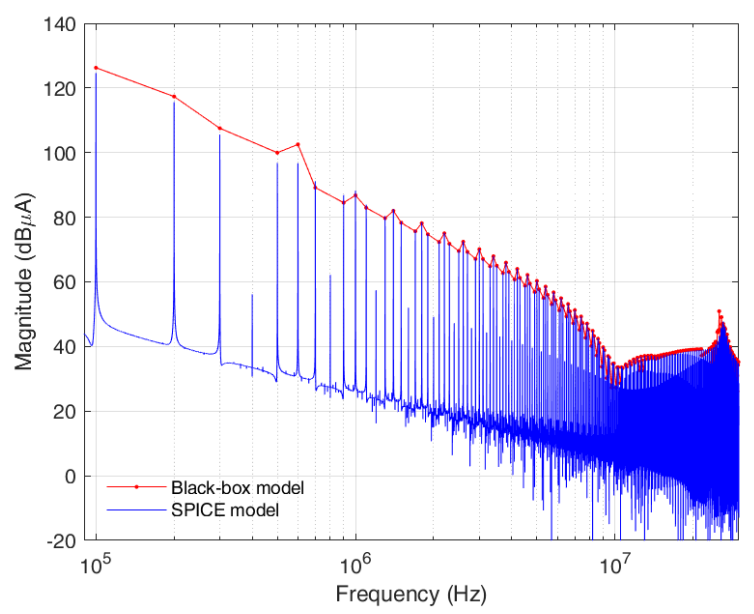

(b)

Fig. 5. Noise current predicted by the black-box model and the circuit (SPICE) model of the boost converter in the presence (a) and in the absence (b) of the input capacitor(s).

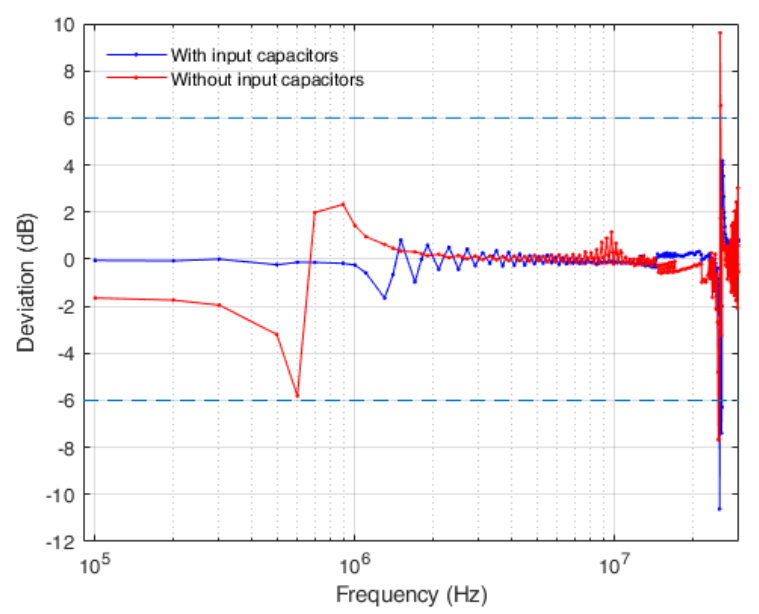

Fig. 6. Deviations between the predictions obtained by the black-box model and the circuit (SPICE) model of the boost converter in the presence and absence of input capacitor(s).

Although the comparison is satisfactory, a general decrease in the prediction accuracy is observed, as it could be better appreciated in Fig. 6, where the differences (in decibels) between the conducted emissions predicted by the SPICE models and those predicted by the corresponding black-box models at $100 \mathrm{kHz}$ and all its harmonics are presented.

The comparison suggests the following conclusions. First, since the largest deviations are observed below $1 \mathrm{MHz}$, it can be inferred that the input capacitor(s) mainly contributes to the masking effect in the lowest frequency range. Second, apart from these deviations, overall the black-box model proves to be able to provide satisfactory prediction of the converter emissions, with the only exception of a narrow interval around $25 \mathrm{MHz}$ due to the resonance already observed also in previous simulations.

This result can be interpreted by recognizing that for the specific boost converter here analyzed, the input capacitor(s) mainly contributes to mask the non-linear and time-variant behavior of the converter at low frequency (i.e., below 1 $\mathrm{MHz}$ ). At high frequency, however, the functional inductor $L=14.7 \mu \mathrm{H}$ mostly contributes to mask the module switching behavior.

\section{ModAl IMPEDANCE ANALYSIS}

Since preliminary measurements proved that the differential mode (DM) impedance of the converter might be sensitive to the operating conditions, the analysis of such an impedance under different operating conditions can provide useful hints on linearity and time-invariant properties of the converter. For instance, if the presence of inductors/capacitors at the converter input can provide a sufficient effect of masking of the non-linear and time-variant behavior associated with the switches, the DM impedance is expected not to exhibit significant deviations by changing the operating conditions. In this respect, the interest here is in investigating possible variations of the DM impedance with the converter switched on and off, since the procedure of derivation of the black-box model foresees to identify the parameters of the passive part with the converter switched off.

For the sake of comparison, both modal impedances will be virtually measured in this section, by adopting the measurement setups shown in Fig. 7. These setups mimic the 


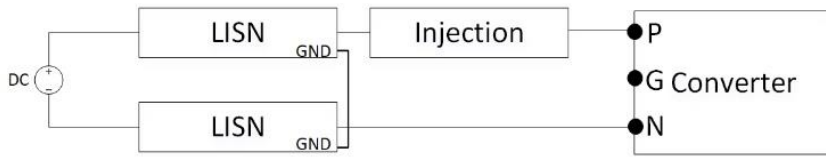

(a)

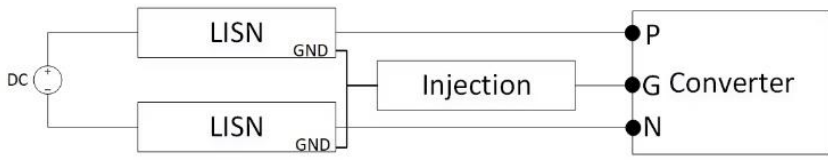

(b)

Fig. 7. Virtual setups for modal impedance measurement: (a) DM; (b) CM.

injection method proposed in [11], where a transformer and an impedance analyzer are adopted in order to measure the model impedances with the converter switched on and under different operating conditions. SPICE modelling of the exploited setups was presented in [12], and applied in this work. CM and DM setups are shown in Fig. 7(a) and (b), respectively, where a series of ideal voltage sources is used to inject the noise at different frequencies. Specifically, since the switching frequency is $100 \mathrm{kHz}$, all harmonics from $100 \mathrm{kHz}$ up to $30 \mathrm{MHz}$ are tested. This allows virtually measuring the $\mathrm{DM}\left(Z_{\mathrm{dm}}\right)$ and $\mathrm{CM}\left(Z_{\mathrm{cm}}\right)$ impedances of the converter both in $\mathrm{ON}$ and $\mathrm{OFF}$ status.

Simulations were run by resorting to the virtual setups shown in Fig. 7 with the boost converter with/without input capacitors. Ideal voltage sources with frequencies $100 \mathrm{kHz}$, $200 \mathrm{kHz}, 300 \mathrm{kHz}, 500 \mathrm{kHz}, 1 \mathrm{MHz}, 2 \mathrm{MHz}, 3 \mathrm{MHz}, 5 \mathrm{MHz}$, $10 \mathrm{MHz}, 20 \mathrm{MHz}, 25 \mathrm{MHz}$, and $30 \mathrm{MHz}$ were connected in series, and used to drive the circuit. Magnitude and phase of the CM impedance evaluated with the boost converter in $\mathrm{ON}$ and OFF status are compared in Fig. 8 both in the presence and absence of the input capacitor(s). As expected, the CM impedance does not exhibit significant differences, as it is mainly dependent on parasitic elements. The corresponding comparison for the DM impedance is shown Fig. 9. Unlike for the CM, for the DM major differences are observed when the input capacitors are present or removed. Since the input capacitors are connected between the phase and neutral lines, connection which naturally affects the DM noise path, the DM impedances evaluated in $\mathrm{ON}$ and OFF status do not exhibit appreciable differences as long as the input capacitor(s) is present. Conversely, when it is removed significant differences can be observed, especially in the frequency range below $1 \mathrm{MHz}$. These results are consistent with those obtained by the previous analysis and documented in Fig. 5 and 6 , where the absence of the input capacitor(s) was causing a decrease in the prediction accuracy of the black-box model approximately below this frequency. Hence, preliminary measurement of the DM impedance in ON and OFF status can provide useful information on the effectiveness of the proposed black-box modelling procedure for the specific converter under analysis. This is especially beneficial when sufficient information about the internal structure of the converter (e.g., on the presence of an effective masking impedance) is not available.

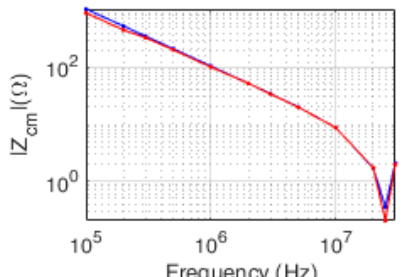

(a)

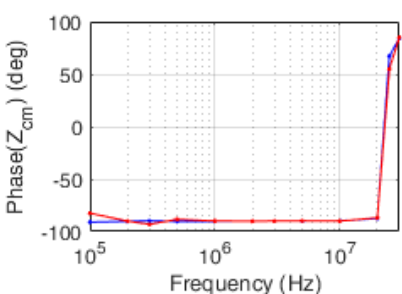

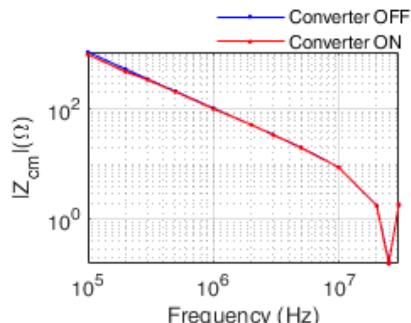

(b)

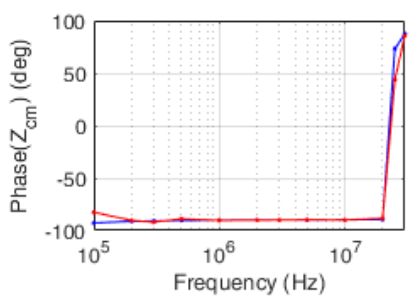

Fig. 8. CM impedance of the boost converter in ON and OFF status: Magnitude (a) with and (b) without input capacitor(s); and Phase (c) with and (d) without input capacitor(s).

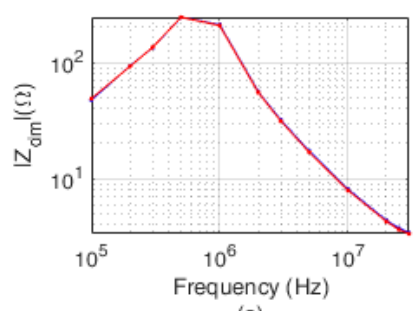

(a)

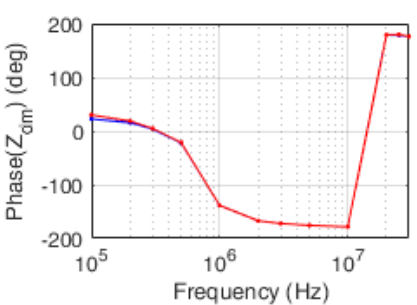

(c)
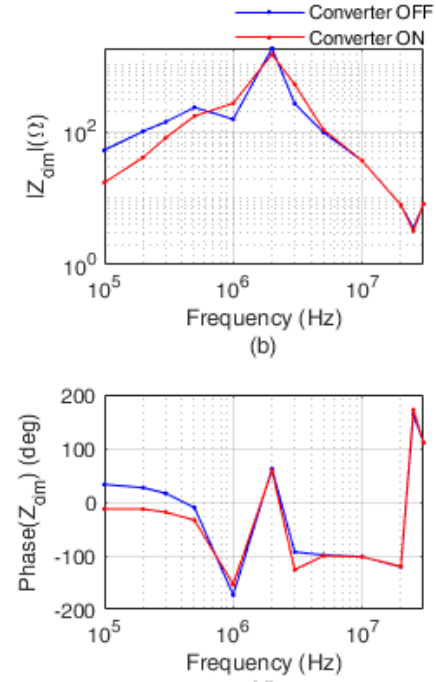

(d)
Fig. 9. DM impedance of the boost converter in $\mathrm{ON}$ and OFF status: Magnitude (a) with and (b) without input capacitor(s); and Phase (c) with and (d) without input capacitor(s).

\section{CONCLUSION}

In this work, suitable conditions for black-box modelling of a boost converter have been investigated by resorting to SPICE simulation of the measurement setups foreseen by the experimental procedure for model-parameter extraction in [6]. More specifically, the analysis focused on the role played by input capacitor(s) and functional inductors, often present in the converter to assure its proper operation, in masking the non-linear and time-variant behavior of the switching modules.

For the boost converter under analysis, it has been proved that the possibility to effectively apply the black-box modelling procedure in [6] is assured mainly thanks to the presence of the functional inductor, while input capacitors play a marginal role, since their effect of masking is appreciable at low frequency only.

Eventually, extension of the analysis to modal impedances allowed identifying the measurement of the DM impedance with the converter in $\mathrm{ON}$ and $\mathrm{OFF}$ status as a useful 
preliminary test to assess whether black-box modelling approaches could be effectively used, even in the absence of detailed information on the converter internal structure. Although for other converter topologies the dominant masking effect can be determined by different circuit components in specific frequency intervals, yet the analysis of the DM impedance under different operating conditions is expected to provide useful information regarding the effectiveness of black-box modeling approaches.

\section{REFERENCES}

[1] G. Rietveld, D. Hoogenboom and M. Acanski, "Conducted EMI Causing Error Readings of Static Electricity Meters," 2018 Conference on Precision Electromagnetic Measurements (CPEM 2018), 2018, pp. $1-2$.

[2] F. A. Kharanaq, A. Emadi and B. Bilgin, "Modeling of Conducted Emissions for EMI Analysis of Power Converters: State-of-the-Art Review," in IEEE Access, vol. 8, pp. 189313-189325, 2020.

[3] Riba J-R, Moreno-Eguilaz M, Bogarra S, Garcia A. Parameter Identification of DC-DC Converters under Steady-State and Transient Conditions Based on White-Box Models. Electronics. 2018; 7(12):393.

[4] G. Frantz, D. Frey, J. -. Schanen and B. Revol, "EMC models of power electronics converters for network analysis," 2013 15th European Conference on Power Electronics and Applications (EPE), 2013, pp. 110.

[5] T. J. Donnelly, S. D. Pekarek, D. R. Fudge and N. Zarate, "Thévenin equivalent circuits for modeling common-mode behavior in power electronic systems," IEEE Open Access Journal of Power and Energy, vol. 7, pp. 163-172, 2020.

[6] G. Spadacini, F. Grassi, D. Bellan, S. A. Pignari, and F. Marliani, "Prediction of conducted emissions in satellite power buses," Int. Journal of Aerospace Eng., vol. 2015, pp. 1-10, Jan. 2015.
[7] B. Sun, R. Burgos and D. Boroyevich, "Common-mode emi unterminated behavioral model of wide-bandgap-based power converters operating at high switching frequency," IEEE Trans. Emerg. Sel. Topics Power Electron., vol. 7, no. 4, pp. 2561-2570, Dec. 2019.

[8] A. Ales, J. Schanen, D. Moussaoui and J. Roudet, "Impedances identification of $\mathrm{dc} / \mathrm{dc}$ converters for network emc analysis," IEEE Trans. Power Electron., vol. 29, no. 12, pp. 6445-6457, Dec. 2014.

[9] H. Bishnoi, A. C. Baisden, P. Mattavelli and D. Boroyevich, "Analysis of emi terminal modeling of switched power converters," IEEE Trans. Power Electron., vol. 27, no. 9, pp. 3924-3933, Sept. 2012.

[10] H. Cheaito, M. Diop, M. Ali, E. Clavel, C. Vollaire and L. Mutel, "Virtual bulk current injection: modeling eut for several setups and quantification of cm-to-dm conversion," IEEE Trans. Electromagn. Compat., vol. 59, no. 3, pp. 835-844, June 2017.

[11] E. Mazzola, F. Grassi and A. Amaducci, "Novel measurement procedure for switched-mode power supply modal impedances," IEEE Trans. on Electromag. Compat., vol. 62, no. 4, pp. 1349-1357, Aug. 2020.

[12] L. Wan, A. Hamid, F. Grassi, G. Spadacini and S. A. Pignari, "SPICE simulation of modal impedances in automotive powertrains under different operating conditions," in Proc. IEEE Int. Symp. Electromagn. Compat., Roma, Italy, Sept. 2020, pp. 1-5.

[13] P. Musznicki, J. -. Schanen, B. Allard and P. J. Chrzan, "Accurate modeling of layout parasitic to forecast EMI emitted from a DC-DC converter," in Proc. 35th Annu. Conf. IEEE Power Electron., Aachen, Germany, Jan. 2004, pp. 278-283.

[14] M. Miloudi, A. Bendaoud, and H. Miloudi, "Common and differential modes of conducted electromagnetic interference in switching power converters Rev. Roum. Sci. Techn.-Électrotechn. Et Énerg vol. 62, no. 3, pp. 246-251, Jul. 2017.

[15] M. Reuter, S. Tenbohlen and W. Köhler, "The influence of network impedance on conducted disturbances within the high-voltage Traction harness of electric vehicles," in IEEE Trans. Electromag. Compat., vol. 56, no. 1, pp. 35-43, Feb. 2014.

[16] R. Hickey, "Extracting scattering parameters from SPICE," TriQuintSemiconductor, Hillsboro, OR, USA, Nov. 1997. 